\title{
Efectos del alcohol en la fisiología humana
}

\author{
Estruch, R.
}

Servicio de Medicina Interna. Hospital Clinic. Barcelona.

Enviar correspondencia: Ramón Estruch. Servei de Medicina Interna. Hospital Clinic. Villarroel 170. 08036 Barcelona. Tel. +34932275400.

E-mail: restruch@clinic.ub.es

\section{Resumen}

El alcohol etílico es tóxico para el organismo, pero consumido de forma esporádica y a dosis bajas, sus efectos son rápidamente reversibles.

Dosis elevadas sobre el tubo digestivo dan lugar a trastornos de la motilidad esofágica, aparición de reflujo gastroesofágico con todas sus posibles consecuencias, gastritis, trastornos del vaciado gástrico y diarreas.

A nivel cardiovascular el consumo agudo da lugar a una reducción aguda de la función contráctil del corazón y a la aparición de arritmias cardíacas. Las alteraciones agudas de la función contráctil del corazón se deben a un efecto inotrópico negativo del etanol sobre el músculo cardíaco, pero este efecto suele quedar enmascarado por un efecto indirecto cronotrópico e inotrópico positivo secundario a la liberación de catecolaminas. Más importancia tiene la relación entre consumo de alcohol y arritmias cardíacas, conocido bajo el término de "corazón del fin de semana" (holiday heart), que debe considerarse como una forma preclínica de miocardiopatía alcohólica.

Además de la embriaguez deben destacarse otros efectos sobre el sistema nervioso central como sus efectos sobre la electrofisiología cerebral (potenciales evocados visuales y auditivos), el metabolismo cerebral y la circulación cerebral.

Existen posibles efectos beneficiosos del consumo de cantidades moderadas como antiséptico gástrico e intestinal, sobre el sistema cardiovascular (reducción de la mortalidad global y de la mortalidad coronaria) y sobre la enfermedad de Alzheimer.

Palabras clave: Alcohol, Fisiología humana, Aparato Digestivo, Sistema Cardiovascular, Presión Arterial, Sistema Nervioso.

\section{Summary}

Ethyl alcohol is toxic for the organism but taken sporadically and at low doses its effects are quickly reversible.

High doses in the digestive tube lead to oesophageal motility disorders, the appearance of gastrooesophogeal reflux with all its possible consequences, gastritis, gastric emptying abnormalities and diarrhoea.

At a cardiovascular level, acute consumption leads to a sharp reduction in the contracting function of the heart and the appearance of cardiac arrhythmias; the former being the result of a negative inotropic effect of the ethanol on the cardiac muscle although this effect is generally masked by an indirect positive chronotropic and inotropic secondary effect from the release of catecholamines. More important is the relationship between alcohol consumption and cardiac arrhythmias, popularly known as "holiday heart" which must be considered as a preclinical form of alcoholic miocardiopathy.

In addition to inebriation, it has other notable effects on the central nervous system on, for example, cerebral electrophysiology (visual and auditory evoked potentials) cerebral metabolism and cerebral circulation.

There are possible beneficial effects from the consumption of moderate quantities as a gastric and intestinal antiseptic, on the cardiovascular system (reduction of overall mortality and of coronary mortality) and on Alzheimer's disease.

Key words: Alcohol, human physiology, digestive apparatus, cardiovascular system, blood pressure, nervous system. 


\section{INTRODUCCIÓN}

E alcohol etílico (etanol) es tóxico para la mayoría de tejidos del organismo. Su consumo crónico y excesivo se ha asociado al desarrollo del síndrome de dependencia al alcohol, pero también a numerosas enfermedades inflamatorias y degenerativas que pueden acabar con la vida de los sujetos que los sufren. El paradigma de las lesiones orgánicas producidas por el consumo crónico de etanol es la cirrosis hepática. Aunque esta enfermedad puede ser debida a otras causas como una infección por los virus de las hepatitis $B$ y $C$, desde hace años se le considera un marcador muy fiable cuanto interesa evaluar y cuantificar a nivel poblacional las lesiones producidas por el consumo crónico de alcohol. No obstante, las lesiones hepáticas por alcohol suelen iniciarse en forma de esteatosis hepática, para progresar posteriormente a una hepatitis alcohólica, una cirrosis hepática e incluso un carcinoma primitivo de hígado. De todos modos, las enfermedades producidas por el consumo crónico de alcohol afectan a casi todos los tejidos y sistemas de organismo. No deben, pues, olvidarse sus efectos sobre el sistema cardiovascular (miocardiopatía alcohólica), páncreas (pancreatitis aguda y crónica), sistema nervioso central (atrofia cerebral y cerebelosa, encefalopatías), nervios periféricos (polineuropatía alcohólica), sistema músculo-esquelético (osteoporosis, miopatía alcohólica) y sobre el feto (síndrome alcohólico fetal), ni tampoco las enfermedades psicoorgánicas (amnesia lacunar, demencia alcohólica), los trastornos psicóticos (alucinosis, celotipia alcohólica) u otras enfermedades psiquiátricas como ansiedad y depresión, a que puede dar lugar. El desarrollo de estas enfermedades depende en gran medida de la cantidad de alcohol consumido por los pacientes (dosis total acumulada de alcohol durante toda la vida del sujeto), aunque también influye una cierta predisposición personal (vulnerabilidad genética) y/o del concurso de determinadas circunstancias ambientales como malnutrición o infecciones concomitantes, como, por ejemplo, los virus de las hepatitis $\mathrm{B}$ y $\mathrm{C}$.
Sin embargo, consumido de forma esporádica, los efectos del etanol sobre la fisiología humana son rápidamente reversibles, por lo que de ningún modo pueden incluirse en el capítulo de enfermedades asociadas a su consumo. Asimismo, consumido a dosis moderadas, aunque sea de forma mantenida, tampoco parecen causar ninguna enfermedad, e incluso se ha sugerido que su consumo a dosis moderadas podría tener efectos beneficiosos sobre la salud. De hecho, estudios realizados durante la última década en países de prácticamente todos los continentes han sugerido que consumo de cantidades bajas y moderadas de alcohol reduce la mortalidad global, pero muy especialmente la mortalidad por causa cardiovascular. En este capítulo se analizan los efectos agudos del etanol sobre los principales sistemas del organismo (digestivo, cardiovascular y nervioso), y también se hace mención de los posibles efectos beneficiosos del consumo moderado de bebidas alcohólicas, especialmente del vino, sobre el organismo.

\section{EFECTOS AGUDOS DEL ETANOL SOBRE EL APARATO DIGESTIVO.}

En la práctica, el primer órgano en que entra en contacto el alcohol es el tubo digestivo, por lo que no debe extrañarnos que afecte directa e indirectamente a un órgano que debe atravesar hasta llegar al torrente circulatorio. Al igual que en los otros tejidos del organismo, los efectos del alcohol sobre el tubo digestivo dependen en gran medida de la dosis administrada, de tal manera que dosis bajas suelen tener muy pocos efectos, mientras que dosis más elevadas dan lugar a una gran variedad de trastornos, como se refiere a continuación (1).

\subsection{Efectos sobre el esófago.}

Numerosos estudios han señalado que la administración aguda y crónica de alcohol, 
tanto por vía oral como intravenosa, da lugar a trastornos de la motilidad esofágica y a la aparición de reflujo gastroesofágico, con todas sus posibles consecuencias.

\subsubsection{Esfínter esofágico inferior (EEI)}

A dosis bajas (30 $\mathrm{g}$ de etanol), el consumo de alcohol no parece tener ningún efecto sobre la motilidad esofágica. En cambio, estudios realizados tanto en sujetos sanos como en alcohólicos crónicos a los que se administró etanol a dosis elevadas $(0,8 \mathrm{~g} / \mathrm{kg}$ de peso) por vía oral e intravenosa han demostrado que el alcohol reduce de forma transitoria la presión basal del EEI, la amplitud de las contracciones de este esfínter y la frecuencia de relajación después de una deglución. Estos efectos resultan ser más intensos en los sujetos sanos (no alcohólicos) que en los pacientes alcohólicos crónicos, lo que sugiere el desarrollo de una cierta tolerancia tras un consumo mantenido de alcohol. Además, desaparecen a las 8 - 24 horas de la administración del alcohol, lo que demuestra su reversibilidad en los sujetos sanos. En los pacientes alcohólicos crónicos, los efectos agudos del alcohol sobre el EEI son diferentes. Tras un primer efecto de reducción de la presión del EEI, se observa un gran aumento de presión en los días siguientes. Esto ha llevado a que algunos autores consideren que parte de los trastornos esofágicos observados en los pacientes alcohólicos crónicos son debidos a un fenómeno de abstinencia (2).

\subsubsection{Motilidad esofágica.}

En sujetos sanos, la administración aguda y crónica de alcohol también reduce la función motora del cuerpo medio del esófago, con reducción tanto de la frecuencia como de la amplitud de las ondas peristálsicas, pero sin afectar a la velocidad de propagación de estas ondas. En cambio, en los alcohólicos crónicos se observa un gran aumento de la amplitud de las ondas peristálsicas. En nuestra experiencia, un $50 \%$ de los alcohólicos crónicos presentan ondas peristálsicas hipercinéticas, con una amplitud de ondas del ter- cio medio del esófago superior a $150 \mathrm{~mm}$ de $\mathrm{Hg}$, lo que define al trastorno como un "esofago en cascanueces" (nutcracker esophagus), junto a una relación entre la amplitud de las ondas entre el tercio medio e inferior del esófago de menos de 0,9 (cuando en todos los controles era superior a 0,9). Esta alteración manométrica explicaría que un elevado porcentaje de pacientes alcohólicos crónicos refieran disfagia leve en el tercio medio del esófago cuando son interrogados específicamente en este sentido (3). Estas alteraciones son reversibles tras varios meses de abstinencia, con reducción de la amplitud de las ondas en el tercio medio del esófago y una normalización de la relación entre la amplitud de las ondas de los tercios medio y distal del esófago. En cambio, las alteraciones persistieron en todos los casos reevaluados que mantenían la ingesta enólica (3).

\subsubsection{Esfínter esofágico superior}

En pocos estudios se ha analizado la función del esfínter esofágico superior, a pesar de que se trata de la última barrera frente a una posible aspiración del material refluido del estómago. La administración aguda de alcohol por vía oral e intravenosa a voluntarios sanos produce una reducción de la presión de este esfínter, junto a una disminución significativa de la amplitud media de contracción tras la deglución. Este trastorno podría explicar, al menos en parte, la elevada incidencia de neumonía por aspiración y de abscesos pulmonares en los pacientes alcohólicos crónicos tras una intoxicación alcohólica aguda. A ello, habría que añadir que cantidades moderadas de alcohol también parecen inhibir el reflejo tusígeno. No obstante, todavía es preciso realizar nuevos estudios en pacientes alcohólicos crónicos con o sin administración de alcohol, para poder llegar a conclusiones definitivas en este sentido.

\subsubsection{Reflujo gastroesofágico}

Por otra parte, el consumo de alcohol facilita el desarrollo de esofagitis por reflujo al reducir tanto la función del esfínter esofágico 
inferior como las contracciones peristálsicas de la porción distal del esófago. La reducción de la presión del esfínter inferior facilita el reflujo del contenido gástrico al esófago, mientras que la disminución de las ondas peristálsicas del esógafo impide una rápida devolución del contenido esofágico al estómago. En un estudio cruzado controlado con placebo se comprobó que la administración de $180 \mathrm{ml}$ de vodka causó una reducción significativa del $\mathrm{pH}$ intraesofágico y un incremento de otros índices de reflujo gastroesofágico comparado con un placebo en 11 de 12 sujetos sanos (4). A partir de estos resultados, los autores concluyeron que la administración de dosis relativamente moderadas de alcohol induce la aparición de reflujo gastroesofágico. En otro estudio a 23 alcohólicos crónicos se les practicó una endoscopia digestiva, una manometría esofágica y un registro del $\mathrm{pH}$ intraesofágico durante 24 horas (3). Más de la mitad de los casos presentaron un reflujo anormal, a pesar de que la presión media del esfínter esofágico inferior estaba aumentada. Ello sólo puede explicarse si el alcohol también altera las relajaciones espontáneas del esfínter, de modo que a lo largo de las 24 horas del día predominan los episodios de trastorno de relajación del esfínter sobre los episodios de hipertensión del mismo. En cualquier caso, los alcohólicos crónicos presentan frecuentemente síntomas de reflujo, alteraciones de la pHmetría de 24 horas y datos histológicos de esofagitis por reflujo.

\subsubsection{Patogenia de los efectos del alcohol sobre el esófago}

La magnitud de estas alteraciones de la motilidad esofágica disminuye de forma significativa tras la administración de alcohol por vía oral e intravenosa, por lo que se ha sugerido que podría tratarse de una manifestación más del síndrome de abstinencia al alcohol. Además, como estos trastornos se observan tanto después de la administración de alcohol por vía oral como por vía intravenosa, no se consideró la posibilidad de que el efecto del etanol fuera directo sobre la musculatura del esófago, sino que se atribuyeron a alteraciones neurológicas. La observación de una mayor prevalencia de estos trastornos de la motilidad esofágica en los pacientes alcohólicos con neuropatía periférica parece confirmar esta hipótesis. No obstante, en un estudio realizado por nuestro grupo se comprobó la presencia de estos trastornos en pacientes alcohólicos tras la ingestión aguda de alcohol. La elevada cifra de alcoholemia de estos pacientes $(1,20 \pm 0,62 \mathrm{~g} / \mathrm{L})$ parece excluir la presencia de una abstinencia enólica. Tampoco se halló ninguna relación de los trastornos de la motilidad esofágica con la presencia de neuropatía periférica definida por criterios electrofisiológicos, ni con la existencia de reflujo gastroesofágico, definido por criterios endoscópicos, histológicos y por pHmetría de 24 horas. Todo ello nos lleva a pensar que se trata de un efecto agudo y directo del etanol sobre la musculatura lisa del esófago. Es conocido que el etanol reduce el contenido de calcio intracitosólico, lo que motiva una reducción de la fuerza de contracción de las fibras musculares lisas. La hipercontractilidad observada en los alcohólicos crónicos debería explicarse por un efecto de liberación de neurotrasmisores, que conduzca a un espasmo de fibras musculares lisas (5).

\subsection{Efectos del etanol sobre el estómago}

Desde hace años se conocen los efectos deletéreos del consumo de etanol sobre la mucosa gástrica. El paradigma de estos efectos es la gastritis aguda inducida por la ingestión de grandes cantidades de alcohol. No obstante, por otra parte, muchos médicos todavía aconsejan el consumo de cantidades moderadas de alcohol como anti-anorexígeno, es decir, para abrir el apetito. El hecho de que predomine uno u otro efecto depende fundamentalmente de la cantidad de alcohol consumida, pero puede que también tengan importancia los productos no-alcohólicos presentes en determinadas bebidas alcohólicas como vino y cerveza. 


\subsubsection{Efectos sobre la función gástrica}

Muchos investigadores han estudiado los efectos del alcohol sobre la secreción ácida gástrica. Estudios en perros con estómagos denervados (sometidos a una vagotomía) han demostrado que la administración tópica de etanol a bajas concentraciones (entre el 8 y $14 \%$ ) produce una estimulación transitoria de la secreción gástrica no mediada por gastrina. En cambio, a concentraciones más elevadas los efectos del etanol son los contrarios, es decir una inhibición de la secreción ácida por el estómago. Estos efectos se acompañaron de alteraciones morfológicas de la mucosa gástrica (véase más adelante). Asimismo, estudios en animales de experimentación han demostrado que dosis bajas de vino y cerveza producían un aumento de la secreción de gastrina e histamina por el estómago, mientras que la administración de la misma cantidad de alcohol en forma de etanol puro no produjo ningún efecto. Estos autores concluyeron que puede que algunos componentes no-alcohólicos de determinadas bebidas sean responsables de los efectos estimulantes de la secreción gástrica (6). Aunque siempre es difícil extrapolar los resultados de estudios realizados en animales de experimentación al hombre, puede que el mencionado efecto estimulante del apetito se deba a esta activación de la secreción ácida, aunque nunca pueden descartarse efectos sobre el hipotálamo.

También se ha referido que el alcohol, a dosis moderadas, tendría un efecto "antiséptico" frente a gérmenes ingeridos por vía oral. Así, en un estudio epidemiológico realizado en el Sudeste de Alemania en el que se incluyeron 1410 adultos entre 15 y 69 años se comprobó que los sujetos que consumían cantidades moderadas de alcohol tenían una menor prevalencia de infección por Helicobacter pylori que las personas abstemias. En cambio, los sujetos que consumían cantidades elevadas de alcohol tenían la misma prevalencia de infección que el grupo control (personas abstemias). El efecto positivo del consumo moderado de alcohol se atribuyó a un posible efecto antiséptico, mientras que la mayor prevalencia de infección por $H$. pylori en los consumidores excesivos de alcohol se explicó por los posible efectos adversos del alcohol sobre el tracto digestivo o sobre el sistema inmune (7).

\subsubsection{Efectos sobre la mucosa gástrica}

La resistencia del estómago a su propia auto-digestión por las enzimas que segrega se debe a la existencia de una barrera gástrica a la difusión del ácido luminal. La primera consecuencia clínica de la alteración de esta barrera es el desarrollo de una gastritis aguda. Estudios en voluntarios sanos han demostrado que tras una intoxicación alcohólica aguda se desarrolla una gastritis endoscópica con eritema, erosiones, petequias y exudado de la mucosa a las 6 horas siguientes en más del $80 \%$ de los casos. Todos estos cambios fueron reversibles en 7 - 20 días en todos los casos en que se repitió la endoscopia. En otro estudio se comprobó que la administración de una dosis única de $200 \mathrm{ml}$ de un licor ya produce un cierto grado de inflamación de la mucosa gástrica, especialmente la del antro y en algunos casos llega a producir erosiones y hemorragias. Estas lesiones habían desaparecido a los tres días en todos los casos reevaluados. La reversibilidad de la gastritis y la propensión a la hemorragia depende del grado de insulto de la barrera de la mucosa gástrica y de la concentración de ácido que entra en contacto con la mucosa lesionada (8). Todavía no se conocen con exactitud cuáles son los mecanismos que utiliza el alcohol para producir las lesiones gástricas, pero los trabajos más recientes sugieren una hiperproducción de factor alfa de necrosis tumoral (TNF- $\alpha$ ), aumento de la apoptosis e incremento de la peroxidación lipídica inducida por radicales libres. Otros irritantes gástricos como la aspirina o los antiinflamatorios no esteroideos pueden potenciar los efectos del alcohol y aumentar el riesgo de hemorragia. No obstante, a pesar de la evidente asociación entre alcohol y lesiones de la mucosa gástrica, no se ha hallado ninguna evidencia concluyente de que el etanol, por si mismo, cause úlcera péptica. 


\subsubsection{Efectos sobre la motilidad gástrica}

La relación entre alcohol y motilidad gástrica es doble. Por una parte, el consumo agudo y crónico de alcohol modifica la motilidad gástrica (vaciado gástrico). Se ha referido que la ingestión de dosis bajas de alcohol no suelen modificar la motilidad gástrica. En cambio, a dosis elevadas, el alcohol disminuye la velocidad de vaciado gástrico. No obstante, por otra parte, se ha comprobado que la absorción del etanol al torrente circulatorio depende en gran medida del vaciado gástrico (9). Un vaciado más rápido del estómago permite el paso de una mayor cantidad de alcohol al intestino y, con ello, una mayor absorción de este tóxico. Ello se explica porque casi todo el metabolismo de primer paso del alcohol tiene lugar en el estómago por la enzima alcohol deshidrogenasa (ADH) de la pared gástrica. Cuanto más rápido sea el paso del alcohol al intestino, más cantidad escapará a la $A D H$ gástrica y la cantidad absorbida será mayor. De hecho, el metabolismo de primer paso del alcohol en los pacientes gastrectomizados es prácticamente nulo, de ahí su mayor vulnerabilidad a los efectos de las bebidas alcohólicas (10).

\subsection{Efectos sobre el intestino delgado}

Un porcentaje relativamente elevado de sujetos presenta diarrea más o menos intensa tras una intoxicación alcohólica aguda. Esta diarrea es de causa multifactorial. Por un lado, la elevada concentración de alcohol en el intestino delgado produce un aumento de la motilidad intestinal. Varios estudios han comprobado que la ingestión aguda de alcohol causa un aumento de la motilidad intestinal, medida, por ejemplo, mediante el tiempo de tránsito oro-cecal. Este efecto se ha atribuido a una acción sobre el sistema nervioso autónomo y/o un efecto inflamatorio sobre la mucosa intestinal (véase más adelante), pero lo más probable es que se trate de un efecto directo del etanol sobre las fibras musculares lisas del intestino delgado (11). Por otro lado, la administración intrayeyunal de alcohol a concentraciones elevadas produce lesiones hemorrágicas de la mucosa intestinal. Asimismo, la administración de concentraciones más fisiológicas (45 - $60 \mathrm{~g}$ de etanol) a voluntarios sanos también produce lesiones estructurales de la mucosa intestinal demostradas por biopsia de yeyuno, junto a una reducción de la actividad de las enzimas disacaridasas de la mucosa intestinal y una alteración en el transporte de fluidos y nutrientes a través de la pared intestinal, con la posibilidad de desarrollo de malabsorción intestinal. En otras palabras, una simple dosis de alcohol ya es capaz de producir lesiones gástricas (antro) y duodenales detectables tanto a nivel macroscópico como microscópico. No obstante, también merece destacarse que el consumo de alcohol aumenta la permeabilidad intestinal incluso antes de que puedan observarse alteraciones de la mucosa intestinal con el microscopio óptico. De hecho, un elevado porcentaje de alcohólicos crónicos presenta malanutrición calórica y/o proteica, aproximadamente un $20 \%$ en nuestro medio (10). Las causas de malnutrición en los alcohólicos son múltiples e incluyen una reducción de la ingesta calórica, una maladigestión de causas gástricas y pancreáticas, malabsorción de los nutrientes en el intestino delgado, insuficiencia hepática (hepatopatía crónica) e incremento de las necesidades metabólicas relacionadas con el propio metabolismo del etanol. Los efectos agudos del alcohol sobre el intestino delgado contribuyen, pues, al menos en parte, en la malnutrición observada en estos pacientes. Curiosamente, en caso de déficits nutritivos, especialmente de ácido fólico y tiamina, se agravan las lesiones producidas por el alcohol en la mucosa intestinal y empeora la malnutrición. No obstante, todos estos efectos son rápidamente reversibles, ya que la diarrea suele ceder a los pocos días de abandonar la ingesta enólica.

\subsection{Efectos agudos sobre el páncreas}

El consumo excesivo de alcohol da lugar a un gran número de alteraciones pancreáticas, entre las que destacan la pancreatitis aguda 
edematosa o necrotizante, y la pancreatitis crónica con o sin insuficiencia pancreática secundaria. No obstante, como no todos los pacientes alcohólicos crónicos desarrollan pancreatitis aguda o crónica, se cree que es necesario el concurso de otros factores para que aparezcan estas enfermedades (véase capítulo 4.1).

Por otra parte, diferentes estudios experimentales han demostrado que el etanol modifica la secreción pancreática tanto in vivo como in vitro. Estos efectos varían según la especie animal analizada y la vía de administración del etanol. En perros y ratas, la administración oral e intragástrica de alcohol estimula la secreción pancreática. Así, la ingestión de etanol aumenta la secreción gástrica, estimula la liberación de secretina y promueve la secreción pancreática a través de varios mecanismos. También en estudios in vitro con acinis pancreáticos extraídos de cobayas se ha comprobado que el etanol induce un incremento de un $50 \%$ en la secreción basal de amilasa, pero inhibe el incremento de la secreción causado por secretina, colecistocinina, carbacol o péptido intestinal vasoactivo (VIP). No obstante, en otros estudios se han referido efectos opuestos, por lo que es posible que esta disparidad de resultados se deba a las diferentes condiciones de los experimentos. En el hombre, se han realizado muy pocos estudios en los que se haya analizado los efectos agudos del etanol sobre la función pancreática. Los resultados han sido muy variables, probablemente debido al empleo de diferentes dosis de alcohol y de distintas bebidas alcohólicas (13). No obstante, la mayoría de autores consideran que el alcohol modifica de forma significativa la secreción pancreática tanto en voluntarios sanos como en alcohólicos crónicos. Los mecanismos a través de los cuáles la administración aguda de alcohol modifica la secreción pancreática no se conocen. En algunos estudios se ha comprobado que el etanol administrado por vía intravenosa aumentaba la secreción de amilasa junto a una elevación de la colecistocinina plasmática. Pero en otros no se ha hallado alteraciones hormona- les, por lo que se ha sugerido que se podría tratar de un efecto directo del etanol sobre el páncreas exocrino (14).

\section{EFECTOS AGUDOS DEL ETANOL SO- BRE EL SISTEMA CARDIOVASCULAR.}

Actualmente nadie duda del efecto deletéreo del consumo excesivo de alcohol sobre el sistema cardiovascular, ya que su consumo excesivo da lugar a patologías graves como la miocardiopatía alcohólica (15), hipertensión arterial (16), arritmias (17) y accidente vasculares cerebrales (18). No obstante, a lo largo de la historia muchos médicos han llegado a prescribir bebidas alcohólicas como "tónico" cardíaco. Asimismo, en los últimos años también han aparecido numerosos artículos que sugieren que el consumo moderado de alcohol reduce la mortalidad global y muy especialmente la mortalidad por cardiopatía coronaria (19). A continuación se analizan los efectos agudos del consumo de alcohol sobre el corazón y la circulación, y posteriormente se comentarán los posibles efectos beneficiosos del consumo moderado de bebidas alcohólicas y especialmente vino sobre el sistema cardiovascular.

En la práctica clínica resulta muy difícil diferenciar los efectos agudos y crónicos del alcohol sobre el sistema vascular, ya que muchas veces ambos efectos se dan simultáneamente en una misma persona. Así, muchos de los efectos "agudos" del alcohol, por ejemplo, sobre el ritmo cardíaco, sólo se observan en pacientes alcohólicos crónicos con una miocardiopatía dilatada, un efecto crónico del consumo de alcohol y, de hecho, en algunas ocasiones los efectos agudos del alcohol sirven para desenmascarar una disfunción cardiaca crónica asintomática (20).

\subsection{Efectos sobre la contractilidad}

Estudios experimentales realizados en miocitos cardíacos o en corazones aislados 
(denervados) han demostrado que el etanol ejerce un efecto inotrópico negativo que conlleva una reducción de la contractilidad cardiaca. Este efecto resulta ser dosis dependiente, ya que cuanto más elevada es la concentración de etanol en la perfusión del corazón, mayor es la depresión de la contractilidad. Además, es rápidamente reversible ya que a los 5 minutos de eliminar el alcohol de la perfusión, el corazón recupera su contractilidad normal (21). Sin embargo, los resultados de la administración in vivo de etanol a animales de experimentación y a humanos han sido muy dispares. Estas discrepancias se deben a que el etanol tiene dos efectos: un efecto directo inotrópico negativo y un efecto indirecto inotrópico y cronotrópico positivo debido a la liberación de catecolaminas. De este modo, el efecto directo negativo sólo puede ponerse de manifiesto si se produce un bloqueo del sistema autónomo. En perros conscientes tratados con propranolol y atropina, la administración aguda de etanol causa una reducción del gasto cardíaco y un incremento de la presión telediastólica (22). Por todo ello, en situaciones normales, el efecto final del etanol sobre la contractilidad cardiaca dependerá de qué acción prevalece.

En la práctica, los efectos agudos del etanol sobre la contractilidad tienen muy pocas consecuencias clínicas tanto en los sujetos no alcohólicos como en los alcohólicos crónicos con función cardiaca normal. Sin embargo, se ha señalado que podrían ser relevantes en los pacientes con miocardiopatía y otros tipos de cardiopatía, ya que se ha señalado que la ingestión de $200 \mathrm{ml}$ de whisky puede producir una reducción significativa del gasto cardíaco y de la fracción de eyección del ventrículo izquierdo en este tipo de pacientes. También se ha referido que la ingestión aguda de alcohol reduce el tiempo de ejercicio hasta la aparición de angina y aumenta la depresión del segmento ST para una misma carga de trabajo. No obstante, no existe un consenso total en este sentido.

Los efectos agudos del etanol sobre la función contráctil del músculo cardíaco son muy pleomórficos. El etanol deprime la contractili- dad al inhibir el proceso de excitación-contracción y al interferir la captación y liberación de $\mathrm{Ca} 2+$ por el sarcolema y retículo sarcoplásmico (véase más adelante) (23).

\subsection{Efectos sobre el ritmo cardíaco}

El consumo de alcohol se ha asociado clásicamente a la inducción de arritmias cardíacas. El etanol tiene un efecto directo sobre el ritmo cardíaco e incluso ingestas relativamente modestas de alcohol dan lugar a una taquicardia rítmica. Por otro lado, los pacientes alcohólicos crónicos presentan frecuentemente enfermedades cardíacas (miocardiopatía), trastornos neuro-hormonales y alteraciones electrolíticas $\left(\mathrm{Na}^{+}, \mathrm{K}^{+}, \mathrm{PO}_{4}^{-}\right.$y $\left.\mathrm{Mg}^{2+}\right)$, que explicaría en parte la elevada frecuencia de arritmias que presentan este tipo de pacientes (17).

La ingesta de cantidades moderadas de alcohol no suelen alterar el ritmo cardíaco en sujetos normales y sólo pocos estudios han observado efectos arritmogénicos de altas dosis de etanol en sujetos no alcohólicos con una función cardiaca normal. Sin embargo, los pacientes alcohólicos crónicos pueden presentar un gran número de arritmias, especialmente tras intoxicaciones agudas de etanol. De hecho, un tercio de los pacientes atendidos en un servicio de Urgencias por una crisis de fibrilación auricular referían una sobreingesta de etanol las horas previas. Asimismo, si sólo se consideran los menores de 65 años, esta proporción aumenta a dos tercios. En este mismo sentido, en un estudio epidemiológico, el riesgo relativo de fibrilación auricular, taquicardia supraventricular y extrasístoles ventriculares era dos veces superior en los sujetos que consumían más de seis copas al día comparado con los que consumían menos de una copa al día (24).

La asociación entre consumo de alcohol y arritmias cardíacas se ha popularizado bajo el término de "corazón del fin de semana" (holiday heart). En 1978, Ettinger et al (25) describieron una serie de 24 pacientes (20 varones y 4 mujeres) que sufrieron arritmias cardíacas 
durante el fin de semana. La arritmia más comúnmente observada fue la fibrilación auricular paroxística, aunque también se observaron otros tipos de arritmia como flutter auricular, taquicardia auricular, taquicardia de la unión, extrasístoles supraventriculares múltiples, y taquicardia ventricular. Aunque ninguno de estos pacientes presentaba datos sugestivos de cardiopatía en un estudio posterior se comprobó que la mayoría de ellos sufría de una miocardiopatía subclínica. Por ello, el síndrome del "corazón del fin de semana debe considerarse como una forma preclínica de la miocardiopatía alcohólica.

En estudios electrofisiológicos se ha comprobado que las arritmias observadas en los pacientes alcohólicos crónicos, principalmente la taquicardia ventricular, pueden desencadenarse por estimulación eléctrica cardiaca tras la ingestión de alcohol, pero no cuando el sujeto se halla sobrio. Estos hechos confirman que la ingestión de alcohol aumentaría la susceptibilidad a presentar arritmias cardíacas en sujetos alcohólicos en los que el consumo de alcohol les provocaba arritmias o palpitaciones. En nuestra experiencia, la aparición de arritmias en los sujetos alcohólicos crónicos depende de la concentración de etanol en plasma (alcoholemia) y de la presencia de miocardiopatía. En un estudio se administró $1 \mathrm{~g}$ de etanol $/ \mathrm{Kg}$ de peso por vía oral a una serie de 20 pacientes con miocardiopatía alcohólica (fracción de eyección inferior al $50 \%$ ), a 20 alcohólicos crónicos con una función cardiaca normal (fracción de eyección superior al $55 \%$ ) y a 10 sujetos sanos. A todos ellos se les realizó un registro electrocardiográfico durante las 8 horas siguientes a la ingestión alcohólica, a la vez que se les determinaba la alcoholemia cada hora. Los pacientes alcohólicos con miocardiopatía presentaron un número significativamente mayor de extrasístoles ventriculares comparado con los alcohólicos sin miocardiopatía y los controles. Además, la mayoría de extrasístoles observados en los alcohólicos con miocardiopatía eran complejos, en forma de dobletes e incluso tripletes. En el grupo de alcohólicos con función miocárdica normal sólo se apreció un aumento significativo de extrasístoles ventriculares simples, comparado con el grupo control. Además, se comprobó que el número máximo de extrasístoles coincidía con el pico máximo de alcoholemia (17). Todos estos hechos explican la elevada prevalencia de muerte súbita tras el consumo de alcohol en los pacientes alcohólicos crónicos.

Los mecanismos implicados en la patogenia de las arritmias cardíacas inducidas por alcohol en los pacientes alcohólicos crónicos parecen ser múltiples. Los alcohólicos crónicos afectos de miocardiopatía clínica o subclínica presentan lesiones focales que pueden retrasar la conducción de estímulos y favorecer la aparición de taquiarritmias por el mecanismo de re-entrada. Los alcohólicos también presentan una prolongación del intervalo QT, que indica una repolarización prolongada que, a su vez, facilitaría la aparición de arritmias ventriculares automáticas. De hecho, la prolongación del intervalo QT es un factor predictivo de mortalidad en los alcohólicos con hepatopatía alcohólica. Los alcohólicos crónicos también presentan una elevación de las catecolaminas circulantes tanto tras una sobreingesta de alcohol como durante las desintoxicaciones. Una estimulación adrenérgica del miocardio puede desencadenar extrasístoles ventriculares y facilitar la aparición de arritmias. De hecho, la hiperactividad simpática se considera una de las principales causas de arritmia en los alcohólicos crónicos. Otras causas de arritmias en los alcohólicos son la presencia de una neuropatía vagal o la existencia de alteraciones electrolíticas como hipopotasemia o hipomagnesemia. Por último, también se han referido las apneas del sueño como causa de arritmias y muerte súbita en este tipo de pacientes.

\subsection{Alcohol y muerte súbita}

Los pacientes alcohólicos tienen un mayor riesgo de sufrir una muerte súbita independientemente de que presenten o no una cardiopatía coronaria. De hecho, aproximada- 
mente una cuarta parte de las muertes súbitas en personas jóvenes o adultas de mediana edad se relacionan con una sobreingesta de alcohol. Muchos de estos casos en realidad sufren una miocardiopatía alcohólica subclínica. Asimismo, en algún estudio epidemiológico se ha observado una correlación entre incidencia de muerte súbita y consumo de alcohol en varones de mediana edad con y sin cardiopatía coronaria. No obstante, en la mayoría de estudios, el aumento en la incidencia de muerte súbita sólo se observaba a partir de seis copas al día (26).

Los mecanismos de muerte súbita relacionados con el consumo de alcohol todavía no se conocen con exactitud. No obstante, como se han identificado pocos pacientes alcohólicos con taquicardias ventriculares sostenidas, se ha postulado que la taquicardia ventricular en los alcohólicos rápidamente degenera a una fibrilación auricular.

\subsection{Efectos del alcohol sobre la función ventricular.}

El consumo excesivo de alcohol tiene un efecto tóxico sobre el miocardio que conlleva la aparición de una miocardiopatía alcohólica. No obstante, el desarrollo de esta entidad no es inmediato, sino que va apareciendo a lo largo de varios años. Se considera que se requiere un consumo de alcohol superior a 10 años para que aparezcan manifestaciones clínicas de una miocardiopatía alcohólica (véase más adelante). Al analizar la historia natural de esta entidad se ha apreciado que primero aparece una disfunción ventricular subclínica (sistólica y/o diastólica) y posteriormente se desarrolla la miocardiopatía alcohólica con signos de insuficiencia cardiaca congestiva y la posibilidad de presentar un gran número de arritmias supra- $y$ ventriculares o incluso una muerte súbita. En los últimos estadios las manifestaciones de esta miocardiopatía son similares a las de la miocardiopatía dilatada idiopática.

De todos modos, la prevalencia de manifestaciones clínicas de insuficiencia cardiaca en los pacientes alcohólicos crónicos es relativamente baja. La mayoría de ellos se hallan asintomáticos durante un largo periodo de tiempo. No obstante, numerosos estudios realizados en este tipo de pacientes han señalado que ya en esta fase de la enfermedad, muchos presentan alteraciones de la función sistólica y/o diastólica del ventrículo izquierdo. En este mismo sentido, estudios necrópsicos de corazones de pacientes alcohólicos crónicos asintomáticos revelan la existencia de aumento del peso cardíaco, dilatación ventricular y cambios histológicos de lesión miocárdica como hipertrofia de miocitos, miocitolisis vacuolar y fibrosis intersticial. Existen, pues, evidencias funcionales e histológicas de lesión miocárdica antes de la aparición de una miocardiopatía alcohólica clínica.

\subsubsection{Disfunción diastólica}

Estudios en animales de experimentación y en pacientes alcohólicos crónicos han referido la existencia de alteraciones de la función diastólica del ventrículo izquierdo tras un consumo crónico y excesivo de alcohol. Así, aproximadamente el $40 \%$ de los varones alcohólicos sin disfunción sistólica, presentan una prolongación del tiempo de relajación ventricular, una reducción de la velocidad máxima diastólica precoz, una menor aceleración del flujo inicial y un mayor cociente aurícula y velocidad máxima precoz, parámetros todos ellos sugestivos de alteración primaria de la relajación miocárdica. También se ha observado una asociación inversa entre función diastólica y consumo moderado de alcohol, lo que sugiere que la fase diastólica del ciclo cardíaco sería más sensible a los efectos tóxicos del alcohol que la fase sistólica y, en consecuencia, que las alteraciones del llenado del ventrículo izquierdo serían una de las primeras manifestaciones de la miocardiopatía alcohólica.

En un estudio realizado en nuestro centro se estudió la función diastólica de 35 alcohólicos crónicos con disfunción sistólica (fracción de eyección $<50 \%$ ) y 77 con función sistólica normal (fracción de eyección > 
$50 \%)$. Un tercio de los pacientes sin miocardiopatía mostraban parámetros ecocardiográficos sugestivos de disfunción diastólica. Asimismo, dos tercios de los pacientes con miocardiopatía (fracción de eyección < 50\%) mostraron también estos parámetros. En cambio, los pacientes con mayor afectación sistólica (fracción de eyección $<32 \%$ ) presentaron una "pseudonormalización de la función diastólica". Asimismo, se halló una correlación entre los parámetros de función diastólica y el consumo de alcohol, independiente de la edad. Se confirma, pues, que la disfunción diastólica parece preceder a la disfunción sistólica en los pacientes alcohólicos crónicos y que está disfunción suele coexistir en los pacientes con disfunción sistólica (miocardiopatía), aunque suele ser más difícil de objetivar en los casos más evolucionados con una fracción de eyección más baja (27).

\subsubsection{Disfunción sistólica}

Asimismo, numerosos estudios en pacientes alcohólicos sin manifestaciones clínicas de insuficiencia cardiaca han referido alteraciones funcionales del ventrículo izquierdo mediante la aplicación de diferentes exploraciones cardiológicas. En un estudio prospectivo realizado por nuestro grupo se analizó la función miocárdica de 46 pacientes alcohólicos a los que se les practicó un estudio cardiológico que incluía ecocardiografía y ventriculografía isotópica. Además, a aquellos en los que se halló una disfunción sistólica del ventrículo izquierdo (fracción de eyección < $50 \%$ ) y dilatación ventricular (diámetro telediastólico $>56 \mathrm{~mm}$ ) se les practicó un cateterismo cardíaco con coronariografía y biopsia endomiocárdica. Los pacientes alcohólicos crónicos presentaron una fracción de eyección significativamente inferior al grupo control (59 frente al 67\%; $p<0,001$ ). Como seis de ellos tuvieron una fracción de eyección inferior al $50 \%$ junto a una dilatación del ventrículo izquierdo, se concluyó que un $13 \%$ de los pacientes alcohólicos crónicos que acuden para desintoxicación presentan una miocardiopatía subclínica. Cuando se analizaron los factores determinantes de esta disfun- ción cardiaca, se comprobó que existía una correlación negativa altamente significativa entre la fracción de eyección y la dosis total acumulada de alcohol $(r=0,63 ; p<0.001)$, así como una correlación positiva entre la masa ventricular izquierda y la dosis total acumulada ( $r=0,54 ; p<0,001)$, por lo que también concluimos que el alcohol es un tóxico para el miocardio de un modo dosis dependiente. De este modo, los alcohólicos que habían bebido más presentaban una mayor afectación cardiaca que los que habían bebido menos cantidad. Asimismo, se halló una dosis umbral de $20 \mathrm{Kg}$ de etanol / $\mathrm{Kg}$ de peso corporal. Aproximadamente un tercio de los pacientes que han bebido más de esta dosis total desarrollan una miocardiopatía subclínica (15).

\subsubsection{Miocardiopatía alcohólica}

La miocardiopatía alcohólica se define como una enfermedad degenerativa del miocardio debida a un consumo excesivo de alcohol, que afecta a personas sin cardiopatía coronaria, hipertensiva, ni valvular. Como no se dispone de ningún marcador específico de esta enfermedad, la relación entre las lesiones miocárdicas y el consumo de alcohol se basa estrictamente en datos epidemiológicos. Los criterios diagnósticos de esta miocardiopatía son: 1) Historia de ingesta prolongada de alcohol, generalmente más de 10 años; 2) Dilatación del ventrículo izquierdo (diámetro telediastólico > $56 \mathrm{~mm}$ o mejor, un índice telediastólico > $31 \mathrm{~mm} / \mathrm{m}^{2}$ ); 3) Fracción de eyección del ventrículo izquierdo baja, generalmente inferior al 50\%); 4) Exclusión de otras causas de cardiopatía. En nuestra experiencia, esta enfermedad se observa típicamente en pacientes alcohólicos entre 30 y 60 años de edad con una ingesta diaria de alcohol entre 112 y $380 \mathrm{~g}$ (media $185 \pm 52$ ) durante un periodo entre 10 y 40 años (media $23 \pm 7$ ). Los síntomas son inespecíficos y similares a otras formas de miocardiopatía dilatada. En cambio, su evolución clínica depende básicamente de su capacidad de abstinencia y del grado de disfunción miocár- 
dica en el momento del diagnóstico (véase más información en el apartado 4.1).

\subsubsection{Fisiopatología de las lesiones cardía- cas inducidas por alcohol}

A pesar de los numerosos estudios realizados, todavía no se conoce con exactitud la fisiopatología de las lesiones cardíacas inducidas por el alcohol. Hace años, las lesiones cardíacas observadas en los pacientes alcohólicos se atribuían a diferentes circunstancias asociadas al alcoholismo crónico. Algunos casos se atribuyeron a contaminaciones de las bebidas alcohólicas por arsénico (epidemia de miocardiopatía en Manchester, Inglaterra). Otra epidemia de miocardiopatías se atribuyó a una intoxicación por cobalto que se utilizaba como antiespumante de la cerveza (casos de Minneapolis en Estados Unidos, Quebec en Canadá y Leuven en Bélgica). También se han referido casos de miocardiopatía por defectos iónicos (P, K, Mg, Se). No obstante, inicialmente la mayoría clínicos e investigadores atribuían la miocardiopatía alcohólica a un déficit de tiamina (vitamina $B_{1}$ ). Sin embargo, existen grandes diferencias clínicas entre la miocardiopatía alcohólica (bajo gasto cardíaco) y el beri-beri occidental (alto gasto cardíaco), la ausencia de mejoría con tratamiento con vitamina $B_{1}$ y la falta de correlación con el estado nutricional de los pacientes. Probablemente, se tratan de circunstancias asociadas al alcoholismo o de casos aislados, pero ninguno de estos factores explica la disfunción ventricular que se observa en un elevado porcentaje de pacientes alcohólicos crónicos.

Actualmente, se cree que las lesiones cardíacas observadas en los alcohólicos crónicos se deben a un efecto tóxico directo del alcohol sobre el miocardio. De hecho, en un estudio realizado en nuestro grupo en una serie de 50 pacientes alcohólicos crónicos se observó una correlación negativa altamente significativa entre la fracción de eyección del ventrículo izquierdo y la dosis total acumulada de alcohol consumida por los pacientes a lo largo de la vida, lo que sugiere un efecto dosis dependiente del alcohol sobre el mio- cardio. Este efecto del etanol se ha relacionado con alteraciones de la respiración mitocondrial y de la oxidación de ácidos grasos similares a las observadas en las mitocondrias del hígado. También se ha señalado que el etanol podría inducir un cambio metabólico del músculo cardíaco hacia un metabolismo glucolítico anaerobio, o causar lesiones por la acción de radicales libres formados por trastornos en la peroxidación de los lípidos o a alteraciones de la síntesis proteica. De todos modos, las teorías más actuales relacionan las alteraciones con cambios en los lípidos y proteínas de las membranas biológicas. Se ha señalado que el etanol altera los canales iónicos, las bombas iónicas e incluso la permeabilidad basal a los iones. Asimismo, estudios in vitro señalan que el etanol reduce la contractilidad muscular a través de mecanismos dependientes del calcio. En los animales expuestos al alcohol se observa una reducción de la densidad de canales de calcio del sarcolema y del retículo sarcoplasmático. También se observa que el etanol interfiere en la unión del calcio a la troponina e incluso en la síntesis de las proteínas contráctiles. Cualesquiera que sea el mecanismo, parece haber un acuerdo en que los cambios en los flujos de calcio intracitosólico son los responsables del efecto inotrópico negativo del etanol. Queda por dilucidar hasta qué punto estos cambios son suficientemente importantes para causar una lesión irreversible de la fibra miocárdica y su posterior necrosis.

Por otra parte, probablemente existan otros factores que junto al consumo de alcohol expliquen por qué sólo una proporción de los pacientes desarrollan una determinada complicación de su alcoholismo. En este sentido se ha señalado que las mujeres son más sensibles que los varones para desarrollar una hepatopatía alcohólica o trastornos cerebrales (atrofia) por alcohol. En nuestro grupo realizamos un trabajo comparativo de los efectos del alcoholismo sobre el funcionalismo cardíaco de 100 varones alcohólicos y 50 mujeres alcohólicas (28). Aunque los varones y mujeres tenían una edad similar, aquéllos habían bebido mucho más que ellas ( $p<$ 
$0,001)$. Además se comprobó que la fracción de eyección de los varones y mujeres alcohólicos se correlacionaba con la dosis total acumulada de alcohol, pero las pendientes de ambas rectas eran significativamente diferentes $(P=0,0006)$. De todo ello se desprende que, a pesar de que las dosis de alcohol ingeridas por las mujeres era sólo el $60 \%$ de la de los varones, la incidencia de miocardiopatía subclínica era similar en uno y otro sexo. Ello, junto a la mayor pendiente de la correlación entre la fracción de eyección y la dosis total acumulada indica que las mujeres son más sensibles que los hombres a los efectos tóxicos del etanol sobre el miocardio.

Por otra parte, actualmente, se está estudiando la relación de determinados polimorfismos de distintos genes en la patogenia de muchas enfermedades. En otro estudio se analizó la participación de otros factores genéticos en el desarrollo de una miocardiopatía alcohólica. Recientemente, se ha sugerido una creciente importancia de la enzima conversora de la angiotensina (ECA) en la hipertrofia cardiaca, funcionalismo miocárdico y mortalidad cardiaca. En este estudio se observó que el $56 \%$ de los alcohólicos con una fracción de eyección inferior al 56\% presentaban el genotipo ECA "DD" comparado con el $8 \%$ de los pacientes con función cardiaca normal. Los pacientes con genotipo DD de la ECA tienen una probabilidad 16 veces superior a los que tienen el alelo "I", lo que indica que también existe una susceptibilidad genética a las lesiones cardíacas por alcohol.

\subsection{Efectos del alcohol sobre la presión arterial}

La relación entre consumo de alcohol y el desarrollo de hipertensión arterial (HTA) es conocida desde hace años al observar una mayor prevalencia de HTA entre los pacientes alcohólicos crónicos. Tanto trabajos de tipo transversal como los escasos estudios longitudinales prospectivos han aportado evidencias de una típica relación de dosis-respuesta entre el consumo de alcohol y la hipertensión arterial cuando la ingestión supera los $30 \mathrm{~g}$ de etanol al día. Los bebedores de menos de esta cantidad presentan cifras tensionales menores a la de los abstemios, lo que confiere una forma de "J" a la relación entre ambas variables (29). Asimismo, en estudios longitudinales como el realizado con 300 controladores aéreos a los que siguieron durante tres años se ha podido constatar un perfecto paralelismo entre las cifras de presión arterial y la ingestión alcohólica. Los sujetos que mantenían la ingestión estable también mantenían estables sus cifras de presión arterial, mientras que aquellos que la aumentaban también incrementaban sus cifras tensionales (30).

El efecto presor del alcohol también se ha estudiado en individuos normotensos e hipertensos. En ambos grupos, la ingestión de alcohol a dosis elevadas (entre 0,75 y $1 \mathrm{~g}$ de etanol/kg de peso) producía una elevación de las presiones sistólica y diastólica, y también de la frecuencia cardiaca. La elevación tensional causada por la ingestión de alcohol varía entre 5 y $8 \mathrm{~mm}$ de $\mathrm{Hg}$ (31-32). En los pacientes alcohólicos crónicos también se ha hallado una prevalencia de HTA superior a la población general y también se ha observado una reducción de las cifras tensionales al cesar su ingesta enólica (16), lo que también confirmaría el efecto presor del alcohol en esta población. Sin embargo, en nuestra experiencia este efecto presor en los pacientes alcohólicos crónicos no es uniforme, ya que sólo se observa en unos pacientes y en otros no. Así, se ha comprobado que aproximadamente el $80 \%$ de los pacientes alcohólicos crónicos hipertensos reducen de forma significativa la presión arterial hasta alcanzar cifras prácticamente normales al mes de abstinencia enólica. En cambio, entre los alcohólicos normotensos, sólo el 50\% muestran una reducción significativa de la presión arterial. Ello nos ha llevado a sugerir la existencia de un grupo de sujetos sensibles a los efectos presores del alcohol y otros resistentes a este efecto, como ocurre con los sujetos sensibles y resistentes a los efectos presores de la sal. 
El efecto presor del alcohol se ha atribuido a una activación del sistema simpático adrenérgico, a un incremento del sistema reninaangiotensina-aldosterona, a un aumento del cortisol, a un incremento de la resistencia a la insulina y/o un vasoespasmo de las fibras músculares lisas de los vasos sanguíneos por alteraciones iónicas $\left(\mathrm{Mg}^{++}\right.$y $\left.\mathrm{Ca}^{++}\right)$o anomalías del transporte de $\mathrm{Na}^{+}$(17).

\subsection{Efectos de dosis moderadas de alco- hol sobre el sistema cardiovascular}

Desde tiempos prácticamente inmemoriales, la sociedad ha atribuido al vino y otras bebidas alcohólicas un efecto beneficioso sobre el sistema cardiovascular, sin ninguna base científica. No obstante, en las últimas décadas numerosos estudios epidemiológicos realizados en múltiples países han demostrado que las curvas de riesgo de mortalidad en función del consumo de alcohol tienen una forma de " $U$ " o de "J", de modo que las personas abstemias tienen un riesgo mayor de muerte que aquellos que beben una cierta cantidad de alcohol, generalmente entre 10 y 40 gramos al día. En el otro extremo de la curva, los pacientes alcohólicos tienen una mortalidad significativamente superior a las personas abstemias y, por supuesto, mayor que aquellas que beben una cantidad reducida de alcohol (33-34).

Estos estudios han recibido gran número de críticas como, por ejemplo, que la mayor mortalidad de las personas abstemias podría ser debida a que en este grupo se hubieran incluido sujetos enfermos en los que se les hubiera prohibido el consumo de alcohol por algún problema médico o incluso podría haber ex alcohólicos. No obstante, estudios más recientes en los que se han controlado estas posibles variables han deparado resultados similares a los anteriores (19). Asimismo, en otros estudios en los que se ha diferenciado el tipo de bebida alcohólica consumida se ha observado que los efectos beneficiosos del vino tinto serían superiores a los de otras bebidas alcohólicas, que se atribuye a sus mayores efectos antioxidantes y antiinflamatorios (35-36).
Incluso, en 1996 la Asociación Americana de Cardiología llegó a concluir que los bebedores moderados de alcohol tienen un riesgo entre el 40 y $50 \%$ menos de sufrir un infarto de miocardio que los abstemios. Existe, pues, un amplio consenso sobre los efectos beneficiosos del consumo moderado de bebidas alcohólicas y del vino en particular sobre la mortalidad global y especialmente sobre la mortalidad coronaria, por lo que actualmente es frecuente que los médicos en general y sobre todo los cardiólogos aconsejen la ingesta moderada de vino a sus pacientes, si no existe una contraindicación formal para ello.

Estos efectos del alcohol sobre el sistema cardiovascular se han atribuido a los siguientes mecanismos:

1. Aumento del HDL-colesterol y especialmente de las subfracciones $\mathrm{HDL}_{2}$ y $\mathrm{HDL}_{3}$.

2. Reducción de la capacidad de oxidación de las partículas de LDL-colesterol.

3. Disminución de la agregabilidad plaquetaria, reducción del fibrinógeno incremento de la actividad fibrinolítica y antitrombina del suero.

4. Cambios en el endotelio vascular que modifica la síntesis de óxido nítrico que causa vasodilatación, y reducción de la síntesis de las moléculas de adhesión monocitarias y endoteliales que participan en los primeros estadios de la arteriosclerosis.

Sin embargo, también se ha referido que la menor mortalidad global y el menor riesgo de presentar una cardiopatía coronaria de los bebedores moderados también podrían ser debido a que este tipo de sujetos tienen hábitos de vida más sanos, como fumar menos, realizar más ejercicio y seguir una dieta mucho más sana. De hecho, el vino es uno más de los múltiples componentes de la dieta Mediterránea, que incluye abundantes frutas y verduras, que contienen una elevada cantidad de compuestos antioxidantes. Puede que la menor mortalidad por cardiopatía coronaria en los países mediterráneos no 
sea debido a su mayor consumo de vino, sino al tipo de dieta que siguen sus habitantes (37). Queda, pues, por establecer de forma definitiva si el consumo moderado de bebidas alcohólicas y especialmente vino tiene realmente un efecto anti-arteriosclerótico o si la menor mortalidad de los bebedores moderados se debe a otros factores concomitantes, como los relacionados con la dieta y el ejercicio.

\section{EFECTOS AGUDOS DEL ALCOHOL SOBRE EL SISTEMA NERVIOSO}

Entre todos los efectos producidos por la ingestión aguda de alcohol sobre el sistema nervioso central merece destacarse la intoxicación alcohólica aguda. Los signos y síntomas de esta intoxicación son bien conocidos y se correlacionan con las concentraciones de alcohol alcanzadas en sangre, que al fin y al cabo traducen los niveles de etanol en el cerebro. A medida que van aumentando la cifra de alcoholemia, el sujeto intoxicado presenta primero una fase de hiperexcitabilidad del córtex, para presentar después un síndrome confusional y cerebeloso, hasta llegar finalmente a sufrir un coma más o menos profundo. También merecen comentarse las intoxicaciones patológicas y las lagunas o amnesias alcohólicas (black outs). La intoxicación patológica se refiere a la rápida aparición de una excitación extrema, con conductas irracionales o violentas, después de la ingestión de pequeñas cantidades de alcohol. Estos episodios duran de minutos a horas y suelen ir seguidos de un estado de somnolencia, del cual el paciente se despierta sin recordar nada. Por algún motivo no conocido el etanol produce un efecto excitante en lugar de sedante, de forma similar a las reacciones patológicas observadas en los pacientes tratados con barbitúricos. Las lagunas alcohólicas se refieren a las amnesias, en ocasiones de horas, que algunos pacientes presentan tras una intoxicación alcohólica aguda. Parece tratarse de un trastorno agudo de la memoria de fijación, sin alteración de las otras funciones cerebrales como la memoria inmediata o la remota. Aunque se le considera como un signo de dependencia al alcohol, las lagunas alcohólicas pueden aparecer en cualquier fase del alcoholismo, incluso en los bebedores esporádicos. En el apartado 4.2 se expone una descripción más detallada de las manifestaciones clínicas de la intoxicación alcohólica aguda, su relación con los niveles de alcoholemia, la fisiopatología de las alteraciones observadas y las principales recomendaciones terapéuticas.

A continuación se exponen efectos menos conocidos del alcohol sobre el sistema nervioso, como son sus efectos sobre la electrofisiología cerebral, el metabolismo neuronal y la circulación del cerebro, junto a los efectos de dosis moderadas de alcohol sobre algunas funciones y alteraciones cerebrales.

\subsection{Efectos del alcohol sobre la electrofi- siología cerebral}

Los efectos del alcohol sobre la actividad eléctrica del córtex cerebral y estructuras profundas del cerebro se han estudiado desde hace años. Aunque existen diferentes métodos de registro de la actividad eléctrica cerebral, los estudios más recientes se han basado en el análisis de los efectos del alcohol sobre los potenciales evocados visuales y auditivos.

Los efectos de dosis bajas de alcohol sobre los potenciales evocados visuales suelen ser mínimos y de hecho en un estudio reciente se comprobaron más alteraciones en el examen de la función cerebral mediante el Wisconsin Card Sorting Test en los sujetos evaluados que trastornos de los potenciales evocados visuales (38). En cambio, en un estudio practicado con 40 alcohólicos asintomáticos que habían acudido para desintoxicación se comprobó una prolongación de la latencia P100 de los potenciales evocados auditivos. Esta alteración estaba muy estrechamente relacionada con la dosis total acumulada de alcohol, de modo que los pacientes que habían bebido más presentaron una alteración significativamente más acusada 
que los que habían bebido menos (39). En cambio, no se apreció ninguna alteración iónica ni nutricional en los pacientes evaluados, por lo que se concluyó que debe tratarse de un efecto tóxico directo del alcohol sobre las vías ópticas.

No obstante, el estudio de los potenciales evocados auditivos ha aportado mucha mayor información. Por un lado, estudios en voluntarios sanos han permitido comprobar como dosis bajas y altas de alcohol alteran los potenciales evocados auditivos, pero esta afectación es diferente según el sujeto tenga o no un alto riesgo de desarrollar un alcoholismo. En efecto, los hijos de pacientes alcohólicos, que tienen un mayor riesgo de desarrollar un alcoholismo (véase el capítulo 6.4. para mayor información), presentan un mayor decremento de la amplitud la onda P200 cuando aumenta la alcoholemia (sensibilidad aguda) y un más rápido retorno a la normalidad de esta onda P200 cuando la alcoholemia disminuye (tolerancia aguda), cuando se compara con sujetos con bajo riesgo de alcoholismo. Estos datos sugieren que los sujetos con alto y bajo riesgo de desarrollar un alcoholismo presentan diferentes sensibilidades a los efectos electrofisiológicos del alcohol (40).

También se han hallado diferencias en los registros de potenciales evocados auditivos entre los pacientes alcohólicos crónicos y diferentes grupos control. Así, se ha comprobado que los alcohólicos crónicos presentan una reducción de amplitud y una prolongación de la latencia de las ondas N100 y P200, junto a una alteración de la prueba del "mismatch negativity". La onda P300 ha despertado gran interés en los investigadores, porque es la que mejor se correlaciona con los resultados de las pruebas neuropsicológicas. Los alcohólicos crónicos también presentan una latencia significativamente más prolongada de la onda P300 que sus controles (41). También en estos casos se trataba de enfermos alcohólicos crónicos sin ningún trastorno nutricional, por lo que lo más probable es que se trate de un efecto dosis-dependiente del alcohol sobre el sistema nervioso central (39).

\subsection{Efectos del alcohol sobre el metabolis- mo cerebral.}

También se han estudiado los efectos agudos del etanol sobre el metabolismo cerebral utilizando la tomografía de emisión de positrones (PET). Tras la administración de $0,5 \mathrm{~g}$ de etanol/ $\mathrm{kg}$ de peso a 8 voluntarios sanos se apreció una reducción de la actividad global cerebral con afectación de prácticamente todas las áreas cerebrales (42). No obstante, estudios posteriores han permitido comprobar que el etanol reducía sobre todo la actividad del córtex occipital, mientras que aumentaba en el córtex temporal izquierdo y ganglios basales izquierdos. Este patrón de afectación es similar al observado en los sujetos tratados con benzodiazepinas (lorazepam), lo que explicaría la similitud de los efectos sedantes entre el alcohol y estos fármacos (43).

\subsection{Efectos del alcohol sobre la circulación cerebral.}

La introducción de la técnica del SPECT (single photon emission tomography) ha permitido el estudio del flujo sanguíneo cerebral regional (rCBF) in vivo en diferentes situaciones. Cuando se aplicó esta técnica para estudiar los efectos agudos de la ingestión de etanol sobre la circulación cerebral se obtuvieron resultados dispares, aunque generalmente existe un cierto consenso en que dosis bajas de alcohol causan una vasodilatación con aumento del rCBF, mientras que dosis altas causan una vasoconstricción con una reducción del rCBF. No obstante, estos efectos no actúan por igual en todos los territorios cerebrales. Así, en un estudio con voluntarios sanos, tras la administración de alcohol se observó un aumento de la circulación del lóbulo prefrontal derecho. No obstante, cuando los sujetos eran pretratados con naloxona i.v. no se apreció ningún cambio cir- 
culatorio, por lo que los autores concluyeron que la euforia que ocurre durante la ingestión aguda de etanol se asocia con una activación del córtex prefrontal derecho que podría estar mediada por el sistema opioide endógeno (véase más adelante) (44).

En cambio, en los alcohólicos crónicos el patrón de alteraciones del flujo sanguíneo cerebral es diferente. En un estudio de 40 pacientes alcohólicos crónicos asintomáticos $(42,5 \pm 9,1$ años) se observó una reducción del flujo sanguíneo cerebral global en todos los lóbulos comparado con los controles ( $p<$ 0,001 ), con afectación en el $65 \%$ de ambos lóbulos frontales y en menor proporción de los lóbulos temporales y occipitales. Los pacientes alcohólicos también presentaban una alteración de las pruebas neuropsicológicas frontales, de tal modo que se halló una correlación altamente significativa entre grado de hipoperfusión frontal y alteración de las pruebas neuropsicológicas (pruebas de trazado $A$ y $B)(r=-0,6535 ; p<0,001)$. Estas alteraciones de la perfusión cerebral se observaron tanto mientras los pacientes mantenían la ingestión alcohólica como a los 10 días de abandonar el hábito enólico. En cambio a los 2 meses de abstinencia, la rCBF de los lóbulos frontales se normalizó en todos los casos en que no se apreció atrofia frontal en la tomografia computerizada (TC) cerebral. Asimismo, se observó una correlación negativa entre la perfusión cerebral y el consumo de etanol en el mes previo $(r=-$ 0,6289; $p<0,001$ ), por lo que se consideró que los efectos de alcohol sobre la circulación cerebral son agudos o subagudos. Así, pues, los alcohólicos asintomáticos presentan una hipoperfusión reversible del lóbulo frontal, que se relaciona con la ingestión reciente de alcohol, refleja una alteración de la función de estos lóbulos y es independiente de la presencia de atrofia frontal (45). Cuando en otro estudio se repitieron las exploraciones después de la administración de 150 mg de naltrexona, se apreció una mayor reducción del rCBF a nivel de los ganglios basales y la región temporal izquierda, áreas muy ricas en receptores opioides.
Estos resultados confirman la implicación del sistema opioide endógeno en el síndrome de dependencia al alcohol (46).

\subsection{Efectos de dosis moderadas de alco- hol}

También se han referido algunos efectos beneficiosos del consumo moderado de bebidas alcohólicas sobre algunas funciones y patologías cerebrales. Así, en un estudio epidemiológico en el que se incluyeron más de 3777 varones y mujeres mayores de 65 años de la región de Burdeos, Francia, se comprobó que los bebedores moderados de vino tenían una prevalencia de enfermedad de Alzheimer un $75 \%$ inferior al grupo control (personas abstemias)(47). Asimismo, en otro estudio posterior los mismos autores observaron que los sujetos mayores de 65 años que bebían regularmente cantidades moderadas de vino obtenían unos resultados en las pruebas neuropsicológicas de evaluación de la función mental significativamente mejores que las personas abstemias (48). De todos modos, antes de considerar estos resultados como definitivos, deberían ser reproducidos por otros grupos de investigación, preferiblemente de otras áreas geográficas no tan ligadas con la producción y comercialización de vino.

\section{BIBLIOGRAFÍA}

(1) Burbige EJ, Lewis DR, Halsted CH. Alcohol and gastrointestinal tract. Med Clin North Am 1984; 68: $77-89\left(^{* *}\right)$.

(2) Keshavarzian A, Polepalle C, Iber FL, Durkin M. Esophageal motor disorder in alcoholics: result of alcoholism or withdrawal?. Alcohol Clin Exp Res 1990; 14: 561 - 567.

(3) Grande L, Monforte R, Ros E, Toledo-Pimentel V, Estruch R, Lacima G, Urbano-Márquez A, Pera C. High amplitude contractions in the middle third of the oesophagus: a manometric marker of chronic alcoholism. Gut 1996; 38: 655 - $662\left({ }^{*}\right)$.

(4) Kaufman SE, Kaye MD. Induction of gastrooesophageal reflux by alcohol. Gut 1978; 19: 336 - 338. 
(5) Altura BM, Altura BT. Peripheral and cerebrovascular action of ethanol, acetaldehyde and acetate: relationship to divalent cations. Alcohol Clin Exp Res 1987; 11: 99 - 111.

(6) Intorre L, Bertini S, Luchetti E, Mengozzi G, Crema F. Soldari G. The effect of ethanol, beer and wine on histamine release from dog stomach. Alcohol 1996; 13: 547 - 551.

(7) Brenner H, Bode G, Adler G, Hoffmeister A, Koenig W, Rothenbacher D. Alcohol as a gastric desinfectant?. The complex relationship between alcohol consumption and current Helicobacter pylori infection. Epidemiology 2001; 12: 209 $-214$.

(8) Gottfried EB, Korstein MA, Lieber CS. Alcoholinduced gastric and duodenal lesions in man. Am J Gastroenterol 1978; 70: 587 - 592.

(9) Oneta, C M; Simanowski, U A; Martinez, M; Allali-Hassani, A; Pares, X; Homann, N; Conradt, C; Waldherr, R; Fiehn, W; Coutelle, C; Seitz, H K. First pass metabolism of ethanol is strikingly influenced by the speed of gastric emptying. Gut 1998; 43: 612 - 619.

(10) Caballeria J, Frezza M, Hernández-Muñoz R, DiPalova E, Korstein MA, Baraona E, Lieber CS. Gastric origin of the first-pass metabolism of ethanol in humans: Effect of gastrectomy. Gastroenterology 1989; 97: 1205 - 1209.

(11) Pfeiffer A, Hoge B, Kaess H. Effect of ethanol and commonly ingested alcoholic beverages on gastric emptying and gastrointestinal transit. Clin Investig 1992; 70: 487 - 491.

(12) Estruch R, Nicolas JM, Villegas E, Junqué $A$, Urbano-Márquez A. Relationship between ethanol-related diseases and nutritional status in chronically alcoholic men. Alcohol Alcohol 1993; 28: 543 - 550.

(13) Hajnal F, Flores MC, Valenzuela JE. Pancreatic secretion in chronic alcoholics. Effects of acute alcohol or wine on response to a meal. Dig Dis Sci 1993; 38: 12 - 17.

(14) Saluja AK, Lu L, Yamaguchi Y, Hofbauer B, Runzi M, Dawra R, Bhatia M, Steer ML. A cholecystokinin-releasing factor mediates ethanolinduced stimulation of rat pancreatic secretion. J Clin Invest 1997; 99: 506 - 512.

(15) Urbano-Márquez A, Estruch R, Navarro-López F, Grau JM, Mont LI, Rubin E. The effects of alcoholism on skeletal and cardiac muscle. $\mathrm{N}$ Engl J Med 1989; 320: 409 - $415\left({ }^{*}\right)$.
(16) Aguilera MT, de la Sierra A, Coca A, Estruch R, Fernández-Solà J, Urbano-Márquez A. Effect of alcohol abstinence on blood pressure: assessment by 24-hour ambulatory blood pressure monitoring. Hypertension 1999;33: 653-7.

(17) Estruch R. Efectos cardiovasculares del alcohol. Med Clin (Barc) 1995; 105: 628 - $635\left({ }^{* *}\right)$.

(18) Monforte R, Estruch R, Graus F, Nicolas JM, Urbano-Márquez A. High ethanol consumption as risk factor for intracerebral hemorrhage in young and middle-aged people. Stroke. 1990; 21: 1529-32.

(19) Gronbaek M, Becker U, Johansen D, Gottshau A, Schnohr P, Hein HO, Jensen G, Sorensen TI. Type of alcohol consumed and mortality from all causes, coronary heart disease and cancer. Ann Intern Med 2000; 191: 411 - $419\left({ }^{* *}\right)$.

(20) Greenberg BH, Schutz R, Grunkemeir GL, Griswold $\mathrm{H}$. Acute effects of alcohol in patients with congestive heart failure. Ann Intern Med 1982; 97: 171 - 175.

(21) Schulman SP, Lakatta EG, Weiss RG, Wolff MR, Hano O, Gerstenblith G. Contractile, metabolic and electrophysiologic effects of ethanol in the isolated rat heart. J Mol Cell Cardiol 1991; 23: 417 - 426.

(22) Kelbaek H, Gjorup T, Hartling OJ, Marving J, Christensen NJ, Godtfredsen J. Left ventricular function during alcohol intoxication and autonomic nervous blockade. Am J Cardiol 1987; 59: 685 - 688.

(23) Thomas AP, Rozanski DJ, Renard DC, Rubin E. Effects of ethanol on the contractile function of the heart: a review. Alcohol Clin Exp Res 1994; 18: 121 - 131.

(24) Koskinen P. Kupari M, Leinonen H. Role of alcohol in recurrences of atrial fibrillation in persons < 65 years of age. Am J Cardiol 1990; 66: 954 - 958.

(25) Ettinger PO, Wu CF, De la Cruz C Jr, Weisse $A B$, Ahmed SS, Regan TJ. Arrhythmias and the "Holiday Heart": Alcohol-associated cardiac rhythm disorders. Am Heart J 1978; 95: 555 $562\left({ }^{*}\right)$.

(26) Kupari M, Koskinen P. Alcohol, cardiac arrhythmias and sudden death. Novartis Found Symp 1998; 216: 68 - 79.

(27) Fernández-Solà J, Nicolás JM, Paré JC, Sacanella E, Fatjo F, Cofan M, Estruch R. Diastolic function impairment in alcoholics. Alcohol Clin Exp Res 2000; 24: 1830 - 1835. 
(28) Urbano-Márquez A, Estruch R, Fernández-Solà J, Nicolás JM, Paré JC, Rubin E. The greater risk of alcoholic cardiomyopathy and myopathy in women compared to men. JAMA 1995; 274 : $149-154\left({ }^{*}\right)$.

(29) Gordon T, Kanel WB. Drinking and its relation to smoking, blood pressure and uric acid: The Fragmingham study. Arch Intern Med 1983; 143: 1366 - 1374.

(30) DeFrank RS, Jenkins CD, Rose RM. A longitudinal investigation of the relationship among alcohol consumption, psychosocial factors and blood pressure. Psychosomatic Med 1987; 49: $236-249$

(31) Potter JF, Watson RS, Skan W, Beevers DG. The pressor and metabolic effects of alcohol in normotensive subjects. Hypertension 1986; 8: $625-631$.

(32) Potter JF, Beevers DG. Pressor effects of alcohol in hypertension. Lancet 1984; 1: 119 - 122.

(33) Hart CL, Smith GD, Hole DJ, Hawthorne VM. Alcohol consumption and mortality from all causes, coronary heart disease and stroke: results from a prospective cohort study of Scottish men with 21 years of follow-up. BMJ 1999; 318: 1725 - 1729.

(34) Mukamed KJ, Maclure M, Mueller JE, Sherwood JB, Mittleman MA. Prior alcohol consumption and mortality following acute myocardial infarction. JAMA 2001; 285: 1965 1970.

(35) Renaud SC, Gueguen R, Schenker J, d'Houtaud $A$. Alcohol and mortality in middle-aged men from eastern France. Epidemiology 1998; 9: 184 - 188.

(36) Imhof A, Froehlich M, Brenner $H$, Boeing $H$, Pepys MB, Koenig W. Effect of alcohol consumption on systemic markers of inflammation. Lancet 2001; 357 : 763 - 767.

(37) Hertog MG, Kromhout D, Aravanis C, Blackburn H, Buzina R, Fidanza F, Giampaoli S, Jansen A, Menotti A, Nedeljkovic S. Flavonoid intake and long-term risk of coronary heart disease and cancer in the seven countries study. Arch Intern Med 1995; 155: 381 - 386.

(38) Quintyn JC, Massy J, Quillard M, Brasseur G. Effects of low alcohol consumption on visual evoked potentials, visual field and visual contrast sensitivity. Acta Ophthamol Scand 1999; 77: $23-26$.
(39) Nicolas JM, Estruch R, Salamero M, Orteu N, Fernández-Solà J, Sacanella E, Urbano-Márquez A. Brain impairment in well nourished chronic alcoholics is related to ethanol intake. Ann Neurol 1997; 41: 590 - $598\left({ }^{* *}\right)$.

(40) Cohen HL, Porjesz B, Stimus AT, Begleiter H. Effects of ethanol on temporary recovery of auditory-evoked potentials in individuals at risk for alcoholism. Alcohol Clin Exp Res 1998; 22: $945-953\left({ }^{*}\right)$.

(41) Keenan JP, Freeman PR, Harrell R. The effects of family history, sobriety length and drinking history in younger alcoholics on P300 auditoryevoked potentials. Alcohol Alcohol 1997; 32: 233 - 239.

(42) De Wit H, Metz J, Wagner N, Cooper N. Behavioral and subjective effects of ethanol-relationship to cerebral metabolism using PET. Alcohol Clin Exp Res 1990; 14: 482 - 489.

(43) Wang GJ, Volkow ND, Franceschi D, Fowler JS, Thanos PK, Scherbaum N, Pappas N, Wong CT, Hitzemann RJ, Felder CA. Regional brain metabolism during alcohol intoxication. Alcohol Clin Exp Res 2000; 29: 822 - 829.

(44) Tishonen J, Kuikka J, Hakola P, Paanila J, Airaksinen J, Eronen M, Hallikainen T. Acute ethanolinduced changes in cerebral blood flow. Am J Psychiatry 1995; 152: 1695 - 1696.

(45) Nicolas JM, Catafau AM, Estruch R, Lomeña FJ, Salamero M, Herranz R, Monforte R, Cardenal C, Urbano-Márquez A. Regional cerebral blood flow - SPECT in chronic alcoholism: relation to neuropsychological testing. J Nucl Med 1993;34: $1452-1459\left({ }^{*}\right)$

(46) Catafau AM, Etcheberrigaray A, Pérez de los Cobos V, Estorch M, Guardia J, Flotats A, Berna L, Mari C, Casas M, Carrio I. Regional cerebral blood flow changes in chronic alcoholic patients induced by naltrexone challenge during detoxification. J Nucl Med 1999; 40: 19 $-24$.

(47) Orgogozo JM, Dartigues JF, Lafont S, Letenneur L, Commenges D, Salomon R, Renaud S, Breteler MB. Wine consumption and dementia in the elderly: a prospective community study in the Bordeaux area. Rev Neurol (Paris) 1997; 153. 185 - 192.

(48) Commenges D, Scotet V, Renaud S, JacqminGadda H, Barberger-Gateau P, Dartigues JF. Intake of flavonoids and risk of dementia. Eur J Epidemiol 2000; 16: 357 - 363. 